included: asthma $(\mathrm{n}=507,18 \%)$, hypertension $(\mathrm{n}=337,12 \%)$, hypercholesterolemia $(\mathrm{n}=157,6 \%)$, coronary heart disease $(\mathrm{n}=79,3 \%)$, immunodeficiency $(\mathrm{n}=11,1 \%)$ and kidney disease $(\mathrm{n}=10,1 \%)$. Overall, $61(2 \%)$ influenza cases developed complications, 21 developed pneumonia of which $85 \%$ had chest $\mathrm{x}$-ray performed. One-third of influenza cases with pneumonia or bronchitis had preexisting conditions like asthma (33\%) and hypertension (34\%). Seventy five percent of Influenza A cases with asthma developed bronchitis or pneumonia. The odds of having severe Influenza were higher among patients with underlying hypertension compared to those without hypertension $(\mathrm{OR}=3.8 ; 95 \% \mathrm{CI}: 2.0-7.1)$. Moreover, influenza cases with asthma had a higher odd of developing pneumonia or bronchitis as compared to those without asthma $(\mathrm{OR}=2.3 ; 95 \% \mathrm{CI}: 1.3-4.0)$. While asthma is a recognized risk factor for influenza complications, isolated hypertension is not.

Conclusion. Identifying people at risk for influenza complications provides a guide for clinicians and public health officials to implement preventive measures and improve clinical outcomes.

Disclosures. All Authors: No reported disclosures

\section{Safety and Efficacy of CR6261 in an Influenza A H1N1 Healthy Human} Challenge Model

Alison Han, MD, MS ${ }^{1}$; Lindsay Czajkowski, MSN, NP-C ${ }^{1}$; Luz Angela Rosas, MS, MLS(ASCP) $\mathrm{CM}^{1}$; Adriana Cervantes-Medina, $\mathrm{BS}^{1}$; Yongli Xiao, $\mathrm{PhD}^{2}$; Monica Gouzoulis, BS ${ }^{2}$; Keith Lumbard, $\mathrm{MS}^{3}$; Sally Hunsberger, $\mathrm{PhD}^{2}$; Susan Reed, $\mathrm{BA}, \mathrm{CRC}^{1}$; Rani Athota, $\mathrm{PhD}^{1}$; Holly Baus, RN, $\mathrm{MSN}^{2}$; Amy Lwin, RN, $\mathrm{BSN}^{4}$; Jerald Sadoff, $\mathrm{MD}^{5}$; Jeffery Taubenberger, $\mathrm{MD}, \mathrm{PhD}^{1}$; Matthew J. Memoli, $\mathrm{MD}, \mathrm{MS}^{2} ;{ }^{1} \mathrm{NIH}$, Rockville, MD; ${ }^{2}$ National Institutes of Health, Bethesda, Maryland ${ }^{3}$ Frederick National Laboratory for Cancer Research, Frederick, Maryland; ${ }^{4} J a n s s e n$ Infectious Diseases and Vaccines, Leiden, Zuid-Holland, Netherlands; ${ }^{5}$ Janssen Infectious Diseases and Vaccines, Leiden, Zuid-Holland, The Netherlands, 2333 , Leiden, Zuid-Holland, Netherlands

Session: P-68. Respiratory Infections - Viral

Background. Influenza virus infections cause significant morbidity and mortality during yearly seasonal epidemics and during sporadic pandemics. It is imperative to identify new targets for vaccines and therapeutics. One such target is the relatively conserved stalk region of the influenza A hemagglutinin (HA) surface protein.

Methods. We conducted a randomized, double-blind, Phase II placebo-controlled trial of a monoclonal antibody that targets the HA stalk (CR6261) in a H1N1pdm09 healthy volunteer human challenge model. CR6261 was infused 24 hours after challenge with H1N1pdm09 and the primary efficacy outcome was area under the curve (AUC) of viral shedding.

Results. Between March 2015-May 2018, 104 healthy volunteers were enrolled and randomized with 91 undergoing influenza challenge, of which 49 participants (54\%) received treatment with CR6261 and 42 participants (46\%) received placebo. A mean of $1 \times 10^{6} \mathrm{ng} / \mathrm{mL}$ of serum CR6261 was detected by 24 hours after infusion. Nasal CR6261 levels reached a peak mean of $5.97 \times 10^{2} \mathrm{ng} / \mathrm{ml} 2$ days after infusion. There was no statistically significant difference in the primary outcome measure between the CR6261 group and placebo (median AUC 48.56 and 25.53 respectively, $\mathrm{P}=0.31$ ). The severity of illness was compared between the two groups, and no significant difference was observed in number of symptoms, duration of symptoms, or FLU-PRO scores.

Conclusion. CR6261 had no statistically significant effect on AUC of vira shedding, and no clinically significant effect on overall influenza disease. Preexisting anti-neuraminidase (NA) antibody titers were most predictive of reduced influenza disease. Nasal CR6261 levels were much lower compared to serum, which may be a factor in the limited effect of CR6261 on this upper respiratory infection. These results suggest that a monoclonal anti-stalk approach to prevent or treat influenza infection may have limited efficacy. Future approaches should consider including and evaluating anti-stalk antibodies as part of a multi-faceted strategy rather than as a standalone therapeutic or vaccine strategy.

Funding. This study was funded in part by the intramural program of NIAID, NIH, by the NCI Contract No. 75N910D00024, Task Order No. 75N91019F00130, and through a CRADA with Janssen Infectious Diseases and Vaccines.

Disclosures. Amy Lwin, RN, BSN, Janssen Pharmaceutical Company of J\&J (Employee) Jerald Sadoff, MD, Janssen Pharmaceutical Company of J\&J (Employee)

1523. Seasonal Human Coronavirus Infections Following Allogeneic Hematopoietic Cell Transplantation: Factors Associated With Lower Respiratory Tract Infection

Chikara Ogimi, $\mathrm{MD}^{1}$; Hu Xie, $\mathrm{MS}^{2}$; Alpana Waghmare, $\mathrm{MD}^{3}$; Masumi Ueda, $\mathrm{MD}^{4}$; Kanwaldeep K. Mallhi, $\mathrm{MD}^{4}$; Chris Davis, $\mathrm{MS}^{5}$; Ashley Akramoff, BS, MA ${ }^{1}$ Anthony Mallory, $\mathrm{BS}^{6}$; Keith R. Jerome, $\mathrm{MD}, \mathrm{PhD}^{7}$; Wendy M. Leisenring, $\mathrm{ScD}^{5}$; Janet A. Englund, $\mathrm{MD}^{8}$; Michael Boeckh, MD PhD ${ }^{5}$; ${ }^{1}$ Seattle Children's Hospital, Seattle, WA; ${ }^{2}$ Fred Hutchinson Cancer Research Center; University of Washington, Seattle, Washington; ${ }^{3}$ Seattle Children's Hospital/University of Washington/Fred Hutchinson Cancer Research Center, Seattle, Washington; ${ }^{4}$ University of Washington/Fred Hutchinson Cancer Research Center, Seattle, Washington; ${ }^{5}$ Fred Hutchinson Cancer Research Center, Seattle, Washington; ${ }^{6}$ University of Washington, Seattle, Washington; ${ }^{7}$ Vaccine and Infectious Disease Division, Fred Hutchinson Cancer Research Center, Department of Laboratory Medicine, University of Washington, Seattle, Washington;
${ }^{8}$ Seattle Children's Hospital/Univ. of Washington, Seattle, Washington

Session: P-68. Respiratory Infections - Viral

Background. Proven/probable lower respiratory tract infection (LRTI) caused by seasonal human coronaviruses ( $\mathrm{HCoVs}$ ) is associated with mortality after hematopoietic cell transplantation (HCT). However, risk factors for LRTI and the significance of virologic documentation of lower respiratory tract involvement by bronchoalveolar lavage (BAL) on outcome are not well characterized.

Methods. Patients receiving allogeneic HCT between 4/2008 and 9/2018 with $\mathrm{HCoV}$ (OC43/NL63/HKU1/229E) detected in nasopharyngeal or BAL samples by PCR were retrospectively analyzed. Proven/probable LRTI was defined as having virus detected from a BAL sample with or without new pulmonary infiltrates by chest radiography, respectively. Possible LRTI was defined as having virus detected from an upper respiratory tract sample with new pulmonary infiltrates. We used logistic regression models to evaluate risk factors for LRTI in patients with first documented HCoV infection during pretransplant conditioning or post-HCT. Overall mortality following proven/probable and possible LRTI was compared by the log-rank test.

Results. A total of 297 patients (61 children and 236 adults) developed $\mathrm{HCoV}$ infection as follows: 254 had upper respiratory tract infection (URTI) alone, 18 presented with LRTI, and 25 progressed from URTI to LRTI [median 16 days (range, 2-62 days)]. Multivariable analyses showed that male gender, higher immunodeficiency scoring index, albumin $<3 \mathrm{~g} / \mathrm{dl}$, glucose $>150 \mathrm{mg} / \mathrm{dl}$ and presence of respiratory copathogen at $\mathrm{HCoV}$ diagnosis were associated with the occurrence of LRTI (Figure 1). Patient with proven/probable LRTI $(\mathrm{N}=16)$ had significantly worse survival than those with possible LRTI $(\mathrm{N}=37)(\mathrm{p}=0.006$, Figure 2$)$.

Figure 1.

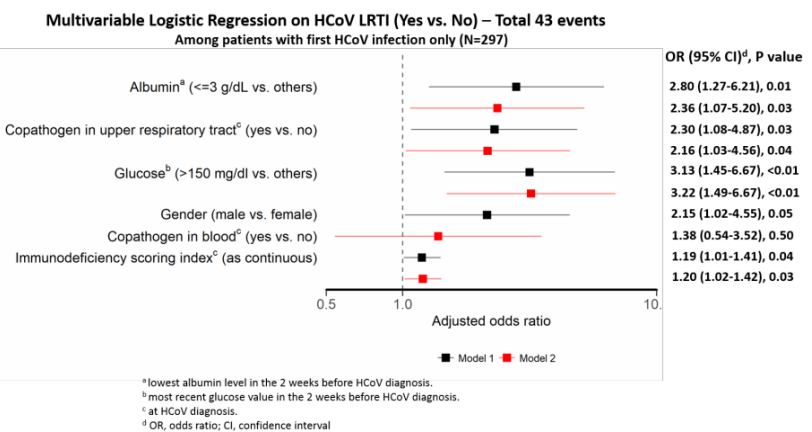

Figure 2.

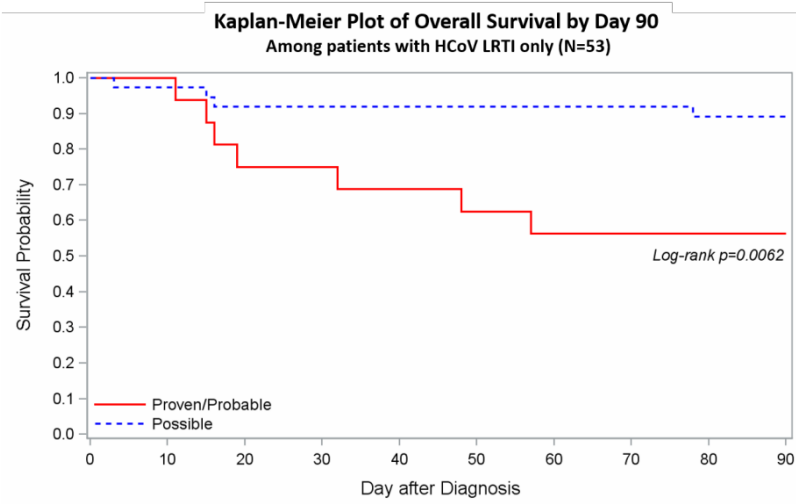

Conclusion. Our analyses identified risk factors (hypoalbuminemia, male gender, high glucose and presence of respiratory copathogen) uncommonly appreciated for LRTI due to other respiratory viruses in HCT recipients. Whether these factors are also relevant to LRTI due to SARS-CoV-2 after HCT requires further studies. The association of hyperglycemia with LRTI might provide an opportunity to reduce the risk of LRTI.

Disclosures. Alpana Waghmare, MD, Amazon (Grant/Research Support)Amazon (Employee, Shareholder)Ansun Biopharma (Scientific Research Study Investigator)Kyorin Pharmaceuticals (Advisor or Review Panel member) Janet A. Englund, MD, AstraZeneca (Scientific Research Study Investigator)GSK group of companies (Scientific Research Study Investigator)Meissa vaccines (Consultant)Merck (Scientific Research Study Investigator)Sanofi Pasteur (Consultant) Michael Boeckh, MD PhD, AlloVir (Consultant)EvrysBio (Advisor or Review Panel member, Other Financial or Material Support, share options)Gilead (Consultant, Grant/Research Support)GSK (Consultant)Helocyte (Advisor or Review Panel member, Shareholder)Lophius (Grant/Research Support)Merck (Consultant, Grant/Research Support)SymBio (Consultant)VirBio (Consultant, Grant/Research Support) 